\title{
Confirmation of the efficacy of hepatic tissue iron index in differentiating genetic haemochromatosis from alcoholic liver disease complicated by alcoholic haemosiderosis
}

\author{
R W Sallie, W D Reed, K B Shilkin
}

\begin{abstract}
The hepatic tissue iron index proposed by Bassett $e t$ al was evaluated in 35 patients with homozygous genetic haemochromatosis, 67 patients with alcoholic liver disease, and 18 patients with other forms of chronic liver disease with and without cirrhosis. In patients with cirrhosis hepatic tissue iron concentration reliably differentiated alcoholic liver disease from genetic haemochromatosis. Although mean iron concentration was greater in patients with prefibrotic haemochromatosis than in those with prefibrotic alcoholic liver disease, some overlap occurred and complete differentiation of the two conditions was not possible. This overlap was particularly evident in some young patients with haemochromatosis in whom the tissue iron concentration grade fell in the range commonly seen in alcoholic haemosiderosis. Inability to differentiate early genetic haemochromatosis from alcoholic liver disease complicated by haemosiderosis was also a problem with standard Perls's staining. When the hepatic tissue iron index was calculated (hepatic tissue iron concentration/patient's age in years), clear differentiation of genetic haemochromatosis from both alcoholic liver disease and other forms of chronic liver disease was obtained in both cirrhotic and precirrhotic patients. This study confirms that the hepatic tissue iron index is a useful means of differentiating patients with genetic haemochromatosis from those with alcoholic liver disease. We suggest that biochemical estimation of tissue iron concentration and calculation of the tissue iron index in all patients in whom genetic haemochromatosis is a possible diagnosis will reduce the likelihood of misdiagnosing this as alcoholic liver disease.
\end{abstract}

Genetic haemochromatosis is a common in herited disorder of iron metabolism. Screening studies of large unselected populations have established a gene frequency of between $6 \%^{1}$ and $10 \%,{ }^{2}$ with a recent Australian study showing a prevalence of the condition of at least $0.29 \% .^{3}$ Untreated genetic haemochromatosis is frequently complicated by primary hepatocellular carcinoma ${ }^{+}$and is therefore one of the most common potentially lethal inherited disorders.

Treatment begun before cirrhosis develops has been shown to return life expectancy to normal..$^{5}$ It is therefore critical that patients with genetic haemochromatosis are identified at the earliest possible stage and treatment started promptly. The high prevalence of alcoholic liver disease in many communities and the familiar finding of iron overload after liver biopsy of patients with alcoholic liver disease ${ }^{56}$ may make it difficult to differentiate genetic haemochromatosis, particularly in young patients, from alcoholic liver disease with histological iron staining alone. Bassett et al have shown the importance of biochemical iron determination and that a tissue iron index, calculated by dividing hepatic tissue iron concentration by the patient's age, is useful in differentiating alcoholic liver disease from genetic haemochromatosis.?

The aim of this study was to examine the efficacy of hepatic tissue iron concentration and the hepatic tissue iron index in differentiating genetic haemochromatosis from alcoholic liver disease.

\section{Patients and methods}

All patients seen in the Gastroenterology/Liver Unit at Sir Charles Gairdner Hospital with genetic haemochromatosis in whom hepatic tissue iron estimation had been performed at diagnosis were studied $(n=35)$. Forty seven patients with genetic haemochromatosis have been identified and are followed up at the hospital, and tissue iron estimation has been performed in all but four diagnosed after 1978 . Eight others without tissue iron estimations were either diagnosed at other institutions, referred to us for follow up, or diagnosed before 1978. The diagnosis of homozygous genetic haemochromatosis was assigned on the basis of immunogenetic data $^{8}$ or if family HLA studies were unavailable or inadequate to assign haplotype by the response to venesection. Probands $(n=14)$ were initially identified by parenchymal iron overload after liver biopsy in the absence of a known cause of secondary iron overload. Approximately $60 \%$ of the first degree relatives of these probands have been screened with serum iron, ferritin, and transferrin saturation and HLA studies, and 21 affected individuals identified. Siblings of a proband with two identical HLA haplotypes were regarded as homozygous for the disorder and advised to undergo liver biopsy. Failure to induce iron deficiency, as judged by microcytic anaemia or low serum ferritin, after six months of weekly venesection was regarded as clinical confirmation of genetic haemochromatosis. Patients with secondary causes of iron overload, such as sideroblastic anaemia and recurrent 
blood transfusion, were excluded from study. Thirty five cases of homozygous genetic haemochromatosis fulfilling these criteria were studied. The diagnosis of alcoholic liver disease was established by a history of excessive alcohol use (>80 g/day men, $>50 \mathrm{~g} /$ day women) for five years or longer and evidence of fatty infiltration or Mallory's hyaline, or both, on liver biopsy specimen.

None of the 67 patients with alcoholic liver disease had a family history of iron storage disorder. Seven patients with alcoholic liver disease and severe iron overload histologically and a tissue iron concentration of $>50 \mathrm{mmol} / \mathrm{kg}$ dry weight underwent weekly venesection, with rapid development of iron deficiency. Eighteen patients with various other forms of chronic liver disease comprising primary biliary cirrhosis $(n=9)$, chronic active hepatitis $(n=4)$, Wilson's disease $(n=2)$, drug induced hepatitis $(n=2)$, and viral hepatitis $(n=1)$, in whom tissue iron had been estimated as part of a diagnostic liver biopsy, were also studied.

\section{LIVER BIOPSIES}

Samples of liver were obtained for histological and biochemical analysis by Trucut liver biopsy. Liver tissue for iron estimation was placed on filter paper and transported in metal-poor normal saline for iron estimation. Liver biopsy specimens were fixed in formalin-saline, stained with haematoxylin and eosin and trichrome and by Perls's Prussian blue stain for histological iron estimation, which was graded $0-4$ based on the modification of Scheur's grading used by Bassett et $a l .^{7}$ According to this, grades 0 (absence of stainable iron) and 1 (stainable iron in less than $25 \%$ of hepatocytes) are normal and grades 2 (stainable iron in $25-50 \%$ of hepatocytes), 3 (75$100 \%$ ), and 4 (heavy iron staining in all hepato- cytes) are abnormal. Otherwise, stained sections were routinely assessed for the presence of architectural distortion, fatty infiltration, Mallory's hyaline, and inflammatory infiltrate.

\section{HEPATIC TISSUE IRON CONCENTRATION}

Hepatic tissue iron was estimated by atomic absorption spectrophotometry. The core of liver tissue, generally $1-2 \mathrm{~cm}$ in length, was dried to constant weight on aluminium foil in an oven at $90^{\circ} \mathrm{C}$, weighed, and digested using a nitric acidhydrogen peroxide mixture. Iron content was then measured by graphite furnace atomic absorption spectroscopy, using an Instrumentation Laboratories IL 655 furnace with Fe hollow cathode lamp set at a wavelength of $372 \mathrm{~nm}$. Using this method the limit of detection of iron is approximately $0.03 \mu \mathrm{mol} / 1$ of digest, or about 0.3 $\mathrm{mmol} / \mathrm{kg}$ dry weight of liver tissue.

The reference range 5-24 mmol Fe/kg dry weight of liver was established from necropsy material taken from 43 patients who died suddenly in road accidents or from other causes. The hepatic histology of these control patients was known to be either normal or show non-specific postmortem changes. The hepatic tissue iron index was calculated by dividing the hepatic tissue iron concentration in $\mathrm{mmol} / \mathrm{kg}$ by the patient's age in years.

\section{STATISTICAL ANALYSIS}

Data are expressed as mean (SD). Hepatic tissue iron concentration is expressed in $\mathrm{mmol} / \mathrm{kg}$ dry weight in liver tissue. Both hepatic tissue iron concentration and tissue iron indices were compared using unpaired Student's $t$ tests. The relation of tissue iron to patient's age was examined for significance using Kendall's tau for non-parametric variables.
Figure 1: Hepatic tissue iron concentration (mmollkg dry weight) related to patient's age. Alcoholic liver disease: prefibrotic $O$, fibroticl cirrhotic O; genetic haemochromatosis: prefibrotic $\square$, fibroticl cirrhotic $\square$; chronic liver disease: prefibrotic $\triangle$, fibroticlcirrhotic $\mathbf{\Delta}$. A significant correlation was found only in patients with genetic haemochromatosis $(r=0.458, p=0.0055$, Kendall's tau).

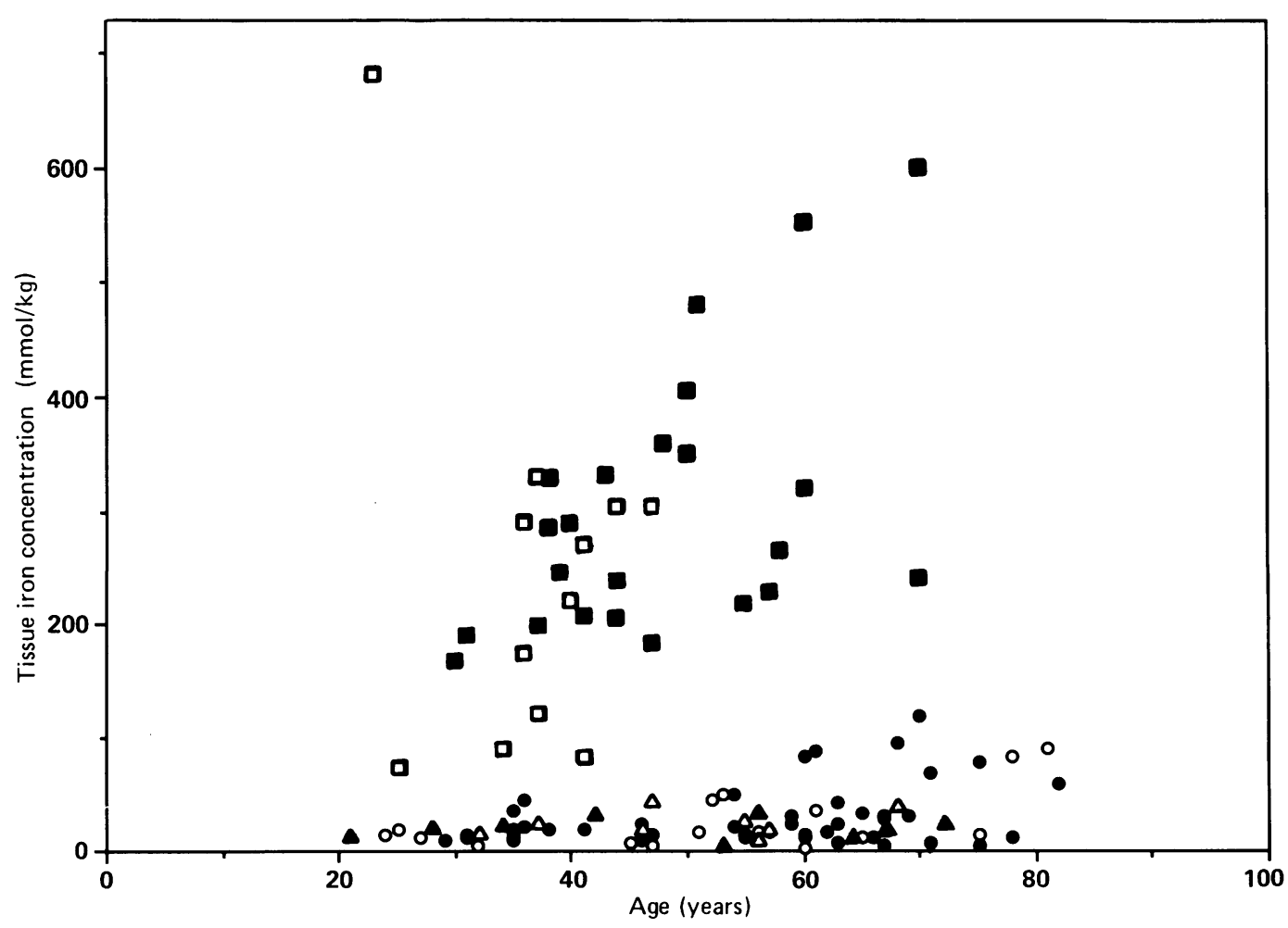


Figure 2: Histological tissue iron grading in patients with alcoholic liver disease $(A L D)$, genetic haemochromatosis $(G H)$, and other forms of chronic liver disease $(C L D)$.

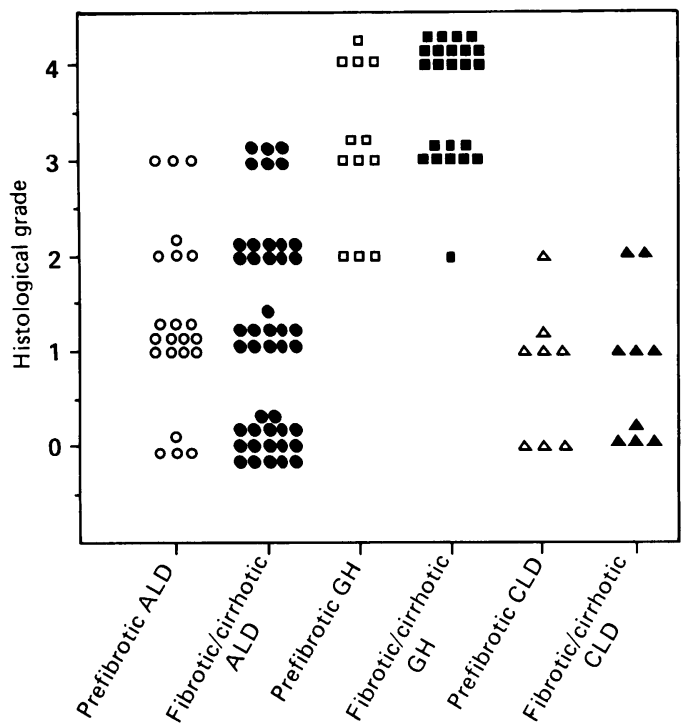

Results

Hepatic tissue iron concentration was found to increase with age in patients with genetic haemochromatosis $(r=0.48, p=0.0055$, Kendall's tau) but not in patients with alcoholic liver disease or other forms of chronic liver disease (Fig 1). Histological iron grading could easily discriminate between patients with cirrhotic genetic haemochromatosis from those with alcoholic liver disease in almost all cases, but was unreliable in differentiating early, prefibrotic genetic haemochromatosis from prefibrotic alcoholic liver disease, particularly in young patients detected at screening (Fig 2). The mean (SD) hepatic tissue iron concentration in patients with prefibrotic alcoholic liver disease $(n=22)$ was $20(21 \cdot 3) \mathrm{mmol} / \mathrm{kg}$ dry weight. This was not significantly different from the concentrations in those with fibrotic or cirrhotic alcoholic liver disease $(n=45)$ at $26 \cdot 8(25 \cdot 3)(p=0 \cdot 3)$, but was significantly less than in patients with prefibrotic genetic haemochromatosis $(n=12)$ at 213 with alcoholic liver disease $(A L D)$, genetic haemochromatosis $(G H)$ and other forms of chronic liver disease (CLD).

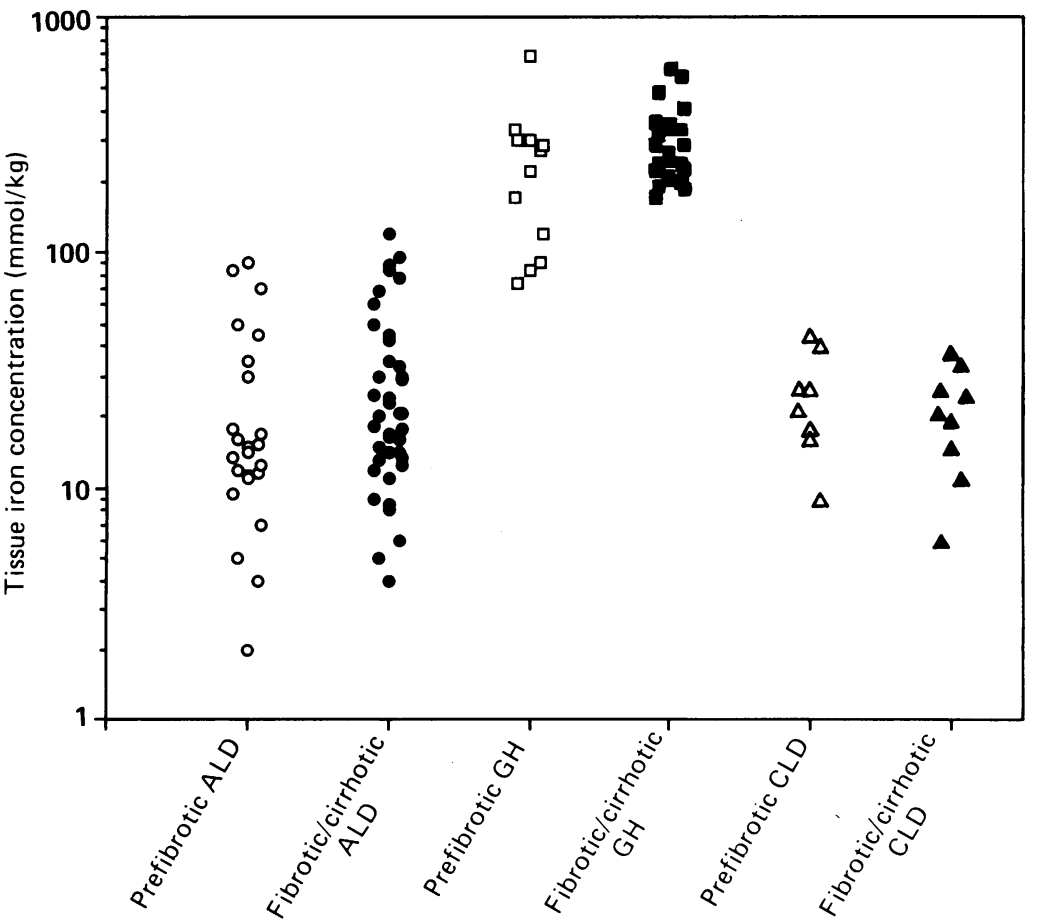

(146.9) $(p=0 \cdot 0005)$. Similarly, patients with fibrotic/cirrhotic alcoholic liver disease had significantly lower hepatic tissue iron concentrations than those with cirrhotic genetic haemochromatosis $(n=23)$ at $315(106)(p=0 \cdot 0067)$. No patient with alcoholic liver disease, with or without cirrhosis, had a hepatic tissue iron concentration above $120 \mathrm{mg} / \mathrm{kg}$ dry weight. Several young homozygous genetic haemochromatosis patients, however, particularly those with normal liver architecture, had hepatic tissue iron concentrations well below this level and, consequently, tissue iron concentration alone was insufficient to completely distinguish prefibrotic alcoholic liver disease and genetic haemochromatosis patients (Fig 3).

When the tissue iron index was calculated a clear differentiation of genetic haemochromatosis from alcoholic liver disease was obtained in both prefibrotic and fibrotic/cirrhotic groups (Fig 4). The mean (SD) hepatic tissue iron index for patients with prefibrotic alcoholic liver disease was $0.439(0.48)$ with the maximum observed 1.65 .

This was not significantly different from cirrhotic alcoholic liver disease (481 (0.42) with a maximum of $1.61(\mathrm{p}=0.715)$, despite the mean age of prefibrotic alcoholic liver disease patients $(50.7$ years) being substantially less than that of fibrotic/cirrhotic patients $(57 \cdot 1$ years $)$ $(\mathrm{p}=0.066))$. The mean hepatic tissue iron index in patients with prefibrotic genetic haemochromatosis was $7 \cdot 58(7 \cdot 21)$, significantly greater than either patients with prefibrotic alcoholic liver disease $(p=0.0067)$ or patients $(n=8)$ with other forms of prefibrotic liver disease $(p=0.0067)$. No patient with alcoholic liver disease had a tissue iron index of $>1.65$, while no patient with genetic haemochromatosis had a tissue iron index of $<2 \cdot 0$. Thus, tissue iron index reliably differentiated patients with alcoholic liver disease from those with genetic haemochromatosis in both cirrhotic and non-cirrhotic cases.

\section{Discussion}

This study confirms the findings of Bassett et al that biochemical measurement of hepatic tissue iron concentration and calculation of the hepatic tissue iron index is valuable in differentiating alcoholic liver disease from genetic haemochromatosis in patients with cirrhosis and in those without cirrhosis. We also confirm that the hepatic tissue iron index is superior to simple biochemical assessment of hepatic tissue iron stores in helping to differentiate genetic haemochromatosis from alcoholic liver disease, particularly in younger patients. This ability to differentiate between the two disorders before architectural distortion occurs makes it particularly valuable in the early diagnosis of genetic haemochromatosis. As Figure 2 shows, histological tissue iron grading is unreliable at differentiating between the two disorders. Some patients in our series had substantial tissue iron concentrations (>100 mmol/kg dry weight liver tissue) despite modest histological evidence of iron overload (grade 2 or 3). This finding is consistent with that reported by Brissot et al. ${ }^{9}$ In their series four of 


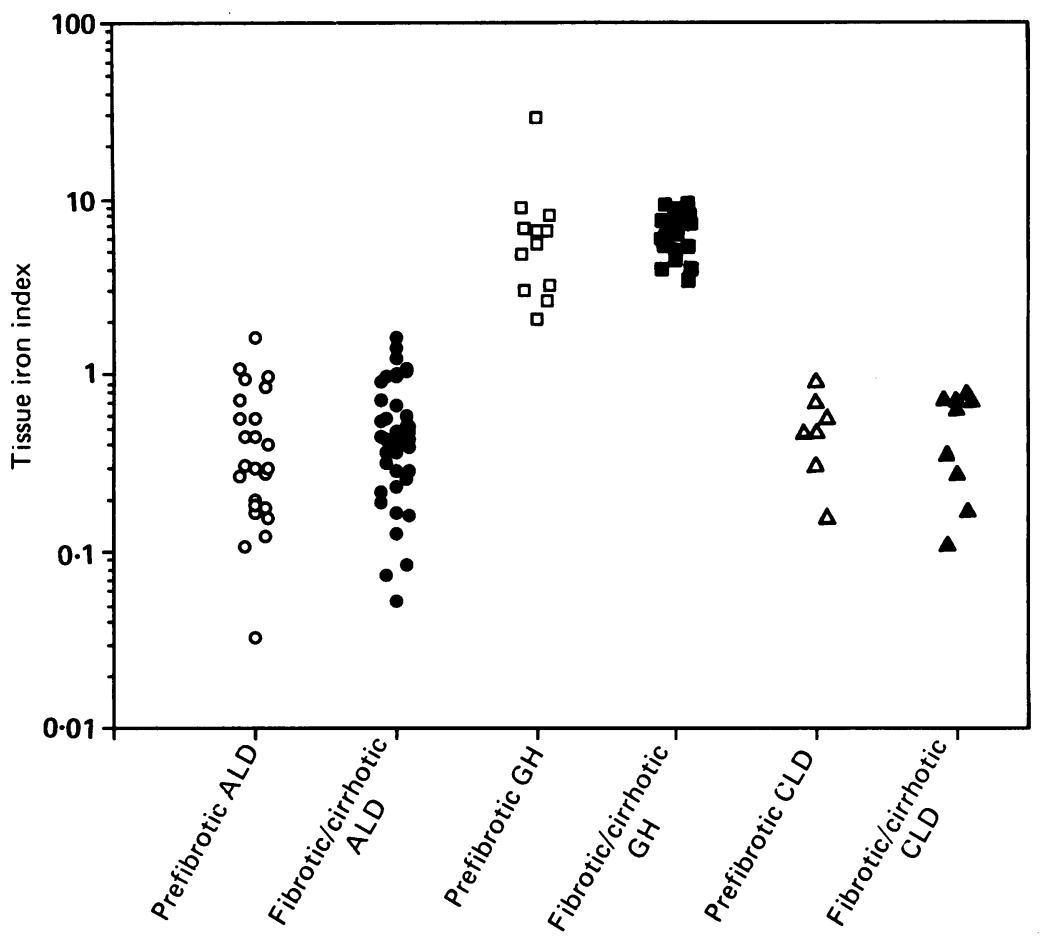

Figure 4: Tissue iron index in patients with alcoholic liver disease ( $A L D)$, genetic haemochromatosis $(G H)$ and other forms of chronic liver disease $(C L D)$.
(>50 mmol $/ \mathrm{kg}$ dry weight) underwent a trial of venesection and rapidly became iron deficient. We were unable to confirm the $400 \mu \mathrm{mol} / \mathrm{g}$ threshold for developing fibrosis/cirrhosis suggested by Bassett $e t a l$ as three of our patients had established cirrhosis with tissue iron concentrations in the range $250-400 \mathrm{mmol} / \mathrm{kg}$ dry weight of liver, in the absence of significant alcohol intake. No patient, however, developed architectural damage at hepatic tissue iron concentrations below $250 \mathrm{mmol} / \mathrm{kg}$ in the absence of alcohol abuse, and this difference between the two studies may also be due to using different methods to measure tissue iron concentration. The highest hepatic tissue iron concentration in this series $(681 \mathrm{mmol} / \mathrm{kg}$ dry weight of liver) was seen in a patient with normal liver architecture despite massive parenchymal iron deposition, and it is possible that tissue damage may be a function of the rate of iron accumulation as well as the absolute level.

This patient presented at age 22 with acute pulmonary oedema secondary to severe cardiomyopathy and died suddenly at age 23 . He had an HLA identical brother, 10 years his senior, with normal iron stores, suggesting recombination between HLA loci and the haemochromatosis gene.

This study confirmed the findings of Bassett et al that the hepatic tissue iron index is a useful means of discriminating between genetic haemochromatosis and alcoholic liver disease complicated by haemosiderosis. We believe that all patients in whom genetic haemochromatosis is a possible diagnosis should have hepatic tissue iron concentration measured and a tissue iron index calculated. These measures will reduce the risk of patients with early genetic haemochromatosis being misdiagnosed as alcoholic liver disease.

We are grateful to Dr Ian Martins who established both the technique of hepatic iron estimation and reference ranges used in this paper while working in the Department of Clinical Biochemistry, Sir Charles Gairdner Hospital, and to MsCathy O'Leary who currently performs the tissue iron estimations. Dr Richard Sallie is the FA Hadley scholar of the University of Western Australia 1988-9 and gratefully acknowledges that support.

1 Edwards C, Griffin L, Goldgar D, Drummond C, Skolnick M, Kushner J. Prevalence of hemochromatosis among 11,065 presumably healthy blood donors. N Engl f Med 1988; 318: presumably

2 Beaumont C, Simon M, Fauchet R. Serum ferritin as a possible marker of the hemochromatosis allele. N Engl $\mathcal{Y}$ Med 1979; 301: 169-74.

3 Leggett B, Halliday J, Bryant S, Powell L. Prevalence of haemochromatosis in an unselected Australian population. Proceedings of the Royal Australasian College of Physicians Golden fubilee Meeting 1988; A51: 395.

4 Strohmeyer G, Niederau C, Stremmel W. Survival and causes of death in hemochromatosis. Observations in 163 patients. Ann NY Acad Sci 1988; 526: 245-57.

5 Niederau C, Fischer R, Sonnenberg A, Stremmel W, Trampisch H, Strohmeyer G. Survival and causes of death in cirrhotic and in non cirrhotic patients with primary hemocirrhotic and in non cirrhotic patients with prim
chromatosis. N Engl f Med 1985; 313: 1256-62.

6 Powell L. Normal human iron storage and its relation to ethanol consumption. Austr Ann Med 1966; 15: 110-15.

7 Bassett M, Halliday J, Powell L. Value of hepatic tissue iron measurement in early hemochromatosis and determination of the critical iron level associated with fibrosis. Hepatology 1986; 6: 24-9.

8 Simon M, Bouyrel M, Genetet B. Idiopathic hemochromatosis and iron overload in alcoholic liver disease: differentiation by HLA phenotype. Gastroenterology 1977; 73: 655-8.

9 Brissot $P$, Bourel $M$, Herry D, et al. Assessment of liver iron content in 271 patients: a reevaluation of direct and indirect methods. Gastroenterology 1981; 80: 557-65. 